\title{
Review of papers presented at the HPTLC Association at the Eastern Analytical Symposium
}

\author{
Fred Rabel ${ }^{1}$
}

Published online: 13 May 2020

(C) Akadémiai Kiadó, Budapest, Hungary 2020

This year, the International Association for the Advancement of High-Performance Thin-Layer Chromatography (HPTLC Association) was allowed to organize and chair a session at the Eastern Analytical Symposium (EAS) meeting, held November 17-19, 2019, at the Crowne Plaza Hotel near Princeton, NJ, USA. The HPTLC Association was organized by researchers and manufacturers in Europe interested in promoting this analytical tool through meetings, an online presence, and new technologies related to TLC/HPTLC. This was also the year they began their North American chapter with this meeting and a special series of papers to be discussed below.

The one-day symposium was organized into two sections. The morning papers were presented by researchers in Europe using HPTLC, while those in the afternoon were by those using HPTLC in North America (NA). The sessions were chaired by Maged Sharad, HPTLC Association. There were about 35 people in attendance throughout the day, many already NA members of the Association.

The first paper, HPTLC Today and the Future, Bernd Spangenberg, University of Applied Sciences, Offenburg, Germany-outlined the use of HPTLC to analyze a complex sample by first separating it into polarity groups via solvent combinations on a silica plate from relative non-polar to more polar eluents, so that each polarity window allows resolution of more peaks. Also, using bonded $\mathrm{CN}$ plates with different solvent combinations, you can be looking at two modes of chromatography, basically, the continued versatility of HPTLC. He spoke about the development in the future of an autorobotic system to perform all the HPTLC steps.

The second paper-Recent contributions of HPTLC to the analysis of complex samples covering wide ranges of polarity - the cases of biofuels, petroleum, and lipidomic analysis-

Fred Rabel

f.rabel@ comcast.net

1 ChromHELP, Woodbury, NJ, USA
Vincente Cebolla, L. Membrado, Spanish National Research Council, C. James, ARID, J. Vela, University of Zaragoza, Spain - outlined the particular difficulty with analysis of these mixtures, but with the use of HPLTC, instrumentation, and special detection techniques, analytical results have been successful. They used AMD (automatic multiple development), thin-layer chromatography-mass spectrometry (TLC-MS), and detection using a method called fluorescence detection by intensity changes. Examples of their successes were shown.

The third paper, The capabilities of effect-directed analysis with HPTLC in environmental analysis, Steven Weiss, Long Distance Water Supply of the State of Baden-Württemberg, Germany - discussed the ongoing nature of continued monitoring of the water supply from the river water used in the district, both before and after treatment. Because of water runoff, the potential contaminants are fungicides, herbicides, and insecticides. They also monitor the water from the waste treatment plant. Using a combination of HPTLC, AMD, MS, sample prep. with 2D HPTLC, and AMD, they are able to see 200 potential impurities. They recently have also begun to use the HILIC mode for the more polar compounds with $\mathrm{AcCN} / \mathrm{H}_{2} \mathrm{O}(8: 2)$ or (5:5) with $250 \mathrm{mM} \mathrm{NaCl}$.

The fourth and final paper of the morning-Comprehensive HPLTC fingerprinting - a new approach to quality, Eike Reich, HPTLC Association, Debora Frommenwiler, CAMAG, Muttenz, Switzerland, S. Canigueral, University of Barcelona, Spain - outlined the progress in getting official organizations to detail HPTLC methods for botanicals in their publications. After many years of standard use for these classes of compounds, the USP added chapters 203 and 1064 and so to in $\mathrm{Ph} \mathrm{Eu} \mathrm{9.0.}$ Examples of analysis of some of these compounds were covered.

After lunch, the fifth paper, HPTLC in the quality control of botanical supplements - identification and beyond, Melissa Daoust, Traditional Medicinals, Rohnert Park, CA, USA, outlined all of their products that are based on the plant materials that they source worldwide, so all the traditional test methods are used to insure the identity and quality of their 
raw materials. Compendial methods because they are vetted are used by her company for all their products, including microscopy and TLC. She went through examples of plant materials they are working with or intending to do so.

The sixth paper, Non-botanical applications of HPTLC, was by Martha Jennes, Eurofins Food Integrity and Innovation, Madison, WI, USA. Their company serves the food industry in many capacities, but she spoke about nonbotanical dietary supplements they test including krill oil, phospholipids, and sucralose, showing the good and bad results from different batches, whether extracted or manufactured.

The seventh paper, HPTLC identification and quantification of cannabinoids in cannabis samples of different chemovarieties, was by Mohamed Radwan, et al., University of Mississippi, MS, USA; Te Do, Eike Reich, Melanie Broszat, and Maged Sharaf, CAMAG, Muttenz, Switzerland. The University of Mississippi is the only US government-approved site for production of cannabis materials for research. All are grown from cuttings to insure genetically pure products. They supply various products made from the plants for research study of any government-approved laboratories. They also analyze samples confiscated by law officials. They noted that the old cannabis products sold in 1995 had a THC content of about $4 \%$, but today that amount reaches $14-16 \%$ because of selective breeding. They produce three general varieties of sample (a) high THC content, (b) THC and CBD combo, and (c) only CBD.

The eighth paper, The validation and transfer of HPTLC methods across multiple laboratories, was by Christopher Lenderink, Amway, Ada, MI, USA. This company manufactures many vitamins and supplements from plant materials, so again HPTLC methods are used worldwide depending on where materials are sourced and/or processed. Moving an an- alytical procedure to other areas of the world is never easy, depending on the method, technology, and training of personnel. Perhaps even more challenging when moving an HPTLC method since this is an open system most often, unless special equipment is being used to lessen variables. He outlined moving methods at this point only between the east and west coasts of America. One method that was transferred did not work out well. They even sent a technician to the west coast facility, and finally pinned it down to a faulty sonicator, not allowing efficient sample extraction. Obviously, this was unexpected, not covered in the method, but only by seeing and hearing one sonicator compared with another found the problem. They also mentioned starting to use DNA analysis of samples to prove authenticity.

The ninth paper, HPTLC in screening for undeclared ingredients, byAnton Bzhelvansky, US Pharmacopeial Convention, Rockville, MD, USA, was withdrawn.

The tenth paper, The International HPTLC Association, by Maged Sharaf, HPTLC Association, introduced the organization's history and purpose to the audience. He outlined ongoing programs. It was founded in 2012 as a nonprofit by a group of European researchers to support HPTLC methods worldwide. There are Association chapters in China, India, Thailand, the UK, and now in North America. See their website for other information, HPTLC methods on plant separations, and how you can join this association to become a part of their learning and sharing of this technology: www. hptlc-association.org

Fred Rabel, $\mathrm{PhD}$

ChromHELP, Woodbury, NJ, USA

Publisher's note Springer Nature remains neutral with regard to jurisdictional claims in published maps and institutional affiliations. 